\title{
Clinical significance of fluorine-18-fluorodeoxyglucose positron emission tomography/computed tomography in the follow-up of colorectal cancer: searching off approaches increasing specificity for detection of recurrence
}

\author{
Semra Ince ${ }^{1}$, Kursat Okuyucu${ }^{1}$, Oguz Hancerliogulları², Engin Alagoz ${ }^{1}$, Huseyin San ${ }^{1}$, \\ Nuri Arslan ${ }^{1}$ \\ ${ }^{1}$ Department of Nuclear Medicine, University of Health Sciences, Gulhane Training and Research Hospital, Ankara, Turkey \\ ${ }^{2}$ Department of General Surgery, University of Health Sciences, Gulhane Training and Research Hospital, Ankara,Turkey
}

Radiol Oncol 2017; 51(4): 378-385.

Received 19 July 2017

Accepted 10 September 2017

Correspondence to: Semra Ince M.D., University of Health Sciences, Gulhane Training and Research Hospital, Department of Nuclear Medicine, 06010 Etlik-Ankara, Turkey. Phone: +90 31230448 28; E-mail: drsemra@gmail.com

Disclosure: No potential conflicts of interest were disclosed.

Background. Nearly $40 \%$ of colorectal cancer (CRC) recurs within 2 years after resection of primary tumor. Imaging with fluorine-18-fluorodeoxyglucose (18F-FDG) positron emission tomography/computed tomography (PET/CT) is the most recent modality and often applied for the evaluation of metastatic spread during the follow-up period. Our goal was to study the diagnostic importance of 18 F-FDG-PET/CT data of maximum standardized uptake value (SUVmax), total lesion glycolysis (TLG) and the difference of SUVmax on dual-time imaging in CRC.

Patients and methods. We examined the SUVmax value of lesions on control or restaging ${ }^{18 F-F D G-P E T / C T ~ o f ~} 53$ CRC patients. All lesions with increased SUVmax values were confirmed by colonoscopy or histopathology. We compared PET/CT results with conventional imaging modalities (CT, MRI) and tumor markers (carbohydrate antigen 19-9 [Ca 199], carcinoembryonic antigen [CEA]).

Results. Mean SUVmax was $6.9 \pm 5.6$ in benign group, $12.7 \pm 6.1$ in malignant group. Mean TLG values of malignant group and benign group were 401 and 148, respectively. 18 F-FDG-PET/CT was truely positive in $48 \%$ of patients with normal Ca 19-9 or CEA levels and truely negative in 10\% of cases with elevated Ca 19-9 or CEA. CT or MRI detected suspicious malignancy in $32 \%$ of the patients and ${ }^{18} \mathrm{~F}$-FDG-PET/CT was truely negative in $35 \%$ of these cases. We found the most important and striking statistical difference of TLG value between the groups with benign and recurrent disease. Conclusions. Although SUVmax is a strong metabolic parameter $(p=0.008)$, TLG seems to be the best predictor in recurrence of CRC $(p=0.001)$; both are increasing the specificity of ${ }^{18}$-FDG-PET/CT.

Key words: metabolic tumor markers; recurrent colorectal cancer; 18F-fluorodeoxyglucose positron emission tomography; computed tomography

\section{Introduction}

Colorectal cancer (CRC) ranks in the third line amongst the most common cancers all over the world. Roundly $40 \%$ of patients recur within 2 years after resection of primary tumor by surgery. ${ }^{1}$ In the follow-up, most guidelines recommend thoracoabdominal CT usually at $12^{\text {th }}, 36^{\text {th }}$ months after surgery or any time in case of clinical doubt as well as routine serial carcinoembryonic antigen 
(CEA) and carbohydrate antigen 19-9 (Ca 19-9) assays. ${ }^{2}$ Imaging has the main role for the evaluation of metastatic spread during the follow-up. Molecular imaging with 18F-fluorodeoxyglucose positron emission tomography combined with computed tomography $\left({ }^{18} \mathrm{~F}-\mathrm{FDG}-\mathrm{PET} / \mathrm{CT}\right)$ is the most recent modality for this purpose. ${ }^{3}{ }^{18} \mathrm{~F}$-FDG$\mathrm{PET} / \mathrm{CT}$ has been used for baseline staging, assessment of treatment response and restaging of CRC as in many other cancers and is concerned to be more sensitive and specific imaging method than routine tools in cases of dubious recurrence and/ or metastasis. 2,3

CEA is expressed by a lot of epithelial tumors and its serum levels may increase in non-malignant conditions such as inflammatory bowel diseases. ${ }^{4}$ Approximately $70 \%$ CRC patients exhibit an elevated CEA level at the time of diagnosis and this fact made it a routine monitoring marker for the disease recurrence. ${ }^{5,6}$ Nevertheless, recent studies of meta-analyses revealed controversies about its utility for the detection of recurrence with a sensitivity of $64 \%$ and a specificity of $90 \%$ which might be considered poor as a biomarker on its own goal. ${ }^{7}$ Ca 19-9 assays have also a poor performance. It has been reported that Ca 19-9 was positive only in 20$40 \%$ of metastatic CRCs. ${ }^{8}$

${ }^{18} \mathrm{~F}-\mathrm{FDG}$-PET has the ability to detect recurrent CRC (as in many other cancers), through pathologically increased tissue metabolism, which precedes the appearance of morphological changes. ${ }^{3,9,10}{ }^{18} \mathrm{~F}$-FDG-PET, however has some intrinsic limitations and its use in the monitoring of CRC is vexed. ${ }^{11,12}$ Latest data offer no indication except the cases with inconclusive CT with suspicion of distant metastasis or in the existence of negative CT and serial CEA rises. ${ }^{13}$ Some current interventions on ${ }^{18} \mathrm{~F}$-FDG-PET/CT such as dual-time or voxelbased dual-time parametric imaging and use of metabolic tumor parameters have been suggested to improve its diagnostic accuracy in several cancers. ${ }^{14}$ Previously, quantitative analyses based on volume-of-interest FDG uptake were introduced. Maximum standardized uptake value (SUVmax) is the vanguard of them. Determination of a cutoff level of SUVmax which differentiates between benign conditions and recurrence of CRC would certainly be helpful. The goal of this paper is to appraise clinical significance of $18 \mathrm{~F}$-fluorodeoxyglucose uptake on FDG-PET/CT in the aftermath of primary curative surgery and/or chemoradiotherapy with respect to recurrence in patients with CRC. We also aimed to research the diagnostic power of ${ }^{18} \mathrm{~F}-\mathrm{FDG}$ PET in recurrent CRC over total lesion glycolysis
(TLG), the difference of SUVmax on dual-time imaging, calculation of a cutoff point of SUVmax discriminating metastasis/recurrence from benign conditions on restaging ${ }^{18} \mathrm{~F}-\mathrm{FDG}-\mathrm{PET} / \mathrm{CT}$.

\section{Patients and methods}

This retrospective cohort study was carried out between 2011 and 2016. It was conducted at nuclear medicine department of a tertiary health care hospital. Inclusion criteria were: histopathologically proven CRC by surgical specimen after primary curative surgery, pathologic FDG uptake on control (evaluation of treatment response) ${ }^{18} \mathrm{~F}-\mathrm{FDG}-$ PET/CT or restaging ${ }^{18} \mathrm{~F}$-FDG-PET/CT performed for the existence of suspicious recurrence or metastasis by routine conventional screening methods in the follow-up, confirmation of all these abnormal uptakes by colonoscopy or histopathologic examination. All cases were treated by surgery and/or chemoradiotherapy. The files of the patients were retrieved from the archive and looked over retrospectively.

We evaluated the lesions on ${ }^{18} \mathrm{~F}-\mathrm{FDG}$-PET/CT in 53 patients. Indications for ${ }^{18} \mathrm{~F}$-FDG-PET/CT were suspicion of recurrence/metastasis (27 patients) and treatment response monitoring (26 patients). Elevated CEA and/or Ca 19-9 levels raised the suspicion of recurrence in 10 cases, conventional imaging (CT or MRI) in 17 cases. All foci of FDG uptake were confirmed by colonoscopic findings and/ or histopathologically. Normal range of Ca 19-9 is $0-35 \mathrm{U} / \mathrm{mL}, \mathrm{CEA}<2.5 \mathrm{ng} / \mathrm{ml}$ for nonsmokers and $<5$ $\mathrm{ng} / \mathrm{ml}$ for smokers.

\section{${ }^{18}$ F-FDG-PET/CT imaging protocol}

$370-555 \mathrm{MBq}$ of ${ }^{18} \mathrm{~F}-\mathrm{FDG}$, calculated according to body weight, was administered to patients by intravenous injection. They fasted for 6 hours prior to the examination and their blood glucose level needed to be below $150 \mathrm{mg} / \mathrm{dl}$ before the injection. Image acquisition was performed 1 hour after the injection with an integrated PET/CT scanner (Discovery 690-GE Healthcare, WI, USA). A lowdose unenhanced CT was performed. CT data were obtained with the automated dose modulation technique of $120 \mathrm{kVp}$ (maximal $100 \mathrm{~mA}$ ), collimated by $64 \times 0.625 \mathrm{~mm}$, measured field of view (FOV) of $50 \mathrm{~cm}$, noise index of $20 \%$ and reconstructed to images of $0.625 \mathrm{~mm}$ transverse pixel size and 3.75 $\mathrm{mm}$ slice thickness. PET emission data were obtained from the middle of thigh up to vertex of the 
skull while the patient was in supine position with the arms rised over head. Acquired PET data were in 3D mode with scanning time of 2 min per bed position and an axial FOV of $153 \mathrm{~mm}$. A standardized way (random, scatter and attenuation) and iterative reconstruction (matrix size $256 \times 256$, Fourier rebinning, VUE Point FX [3D] with 3 iterations, 18 subsets) were used for correction of emission data. Dual-time ${ }^{18} \mathrm{~F}-\mathrm{FDG}$-PET/CT was performed in 28 patients. $105 \pm 10$ minutes post-injection after the completion of standard protocol, delayed imaging for the whole abdomen with the CT scan and repositioning was performed.

\section{Visual and quantitative interpretation}

${ }^{18} \mathrm{~F}-\mathrm{FDG}-\mathrm{PET} / \mathrm{CT}$ images were interpreted visually by two nuclear medicine specialists aware of patient history. Focally or heterogeneously increased FDG uptake, diffuse or heterogeneously increased FDG uptake and/or soft tissue mass on CT component, hipodense or nodular lesion on CT (with or without FDG uptake), diffuse uptake accompanied by wall tickening, consolidation or ambiguous lesions on CT (with or without uptake) were accepted pathologic. SUVmax was calculated for all patients. Other quantitative parameters of average standardized uptake value (SUVmean), metabolic tumor volume (MTV) and TLG were calculated in 20 cases on ${ }^{18} \mathrm{~F}$-FDG-PET/CT. We calculated TLG values by multiplying MTV and SUV mean. The corresponding CT scans of lesions were used as a guideline to demarcate them if their boundaries were difficult to define for the calculation of SUVmax.

Quantitative PET/CT parameters were calculated from a routine protocol used on a sophisticated workstation (Volumetrix for PET-CT and AW volume share 4.5, GE Healthcare, Waukesha, WI, USA). Standard methods computed SUVmax and SUVmean, corrected for body weight, from the voxel having the most intense activity in three-dimensional tumor region on transaxial whole-body images of attenuation-corrected PET/CT images. MTV $\left(\mathrm{cm}^{3}\right)$ was measured with a half-automatic PET analysis computer program, having an automatic isocontour threshold method based on a notion of being greater than $42 \%$ of the SUV max value within the tumor.

\section{Statistical analysis}

The whole data were analysed by IBM Corp. Released 2013. IBM SPSS Statistics for Windows,
TABLE 1. Locations of pathologic FDG uptake

\begin{tabular}{lc}
\hline Sites of abnormal FDG uptake & $\mathbf{n}$ \\
\hline Anostomosis line (area) & 7 \\
Rectum & 9 \\
Rectosigmoid region & 11 \\
Liver & 9 \\
Caecum & 1 \\
Kidney & 1 \\
Abdominal mass & 4 \\
Presacral mass & 5 \\
Sigmoid region & 3 \\
Descending colon & 2 \\
Lung & 1 \\
\hline
\end{tabular}

Version 22.0. Armonk, NY: IBM Corp. Number and percentage values were used for the description of categorical data. Mean, median, standard deviation (SD), minimum and maximum values described continuous data. Intergroup (benign conditions versus malignant group) comparisons were carried out by Mann-Whitney U test for SUVmax, TLG and the difference between SUVmax values of dual-time imaging. Wilcoxon test was used for ingroup comparison between SUVmax values of early and delayed imaging for benign and malignant group. The variables having a value of $p<0.05$ were accepted as statistically meaningful. ROC curve analysis was plotted to see the diagnostic value of SUVmax on recurrent disease. Informed consent was supposed as a retrospective study which permitted to use records, documents and data of patients applied on our clinic for the test. The study was ratified by our Institutional Review Ethics Committee (approval number 80/2016). This study conforms to the Declaration of Helsinki.

\section{Results}

Mean age was $58.6 \pm 10.9$ years (30-89). 24 of the patients were male (45\%), 29 of them were female (55\%). Primary tumor sites were rectum $(n=23)$, sigmoid colon $(n=5)$ and other colonic segments $(\mathrm{n}=25)$. The most common localization of FDG uptake was rectosigmoid region (43.3\%). Locations of pathologic FDG uptake were demonstrated in Table 1. Sensitivity, specificity, positive predictive value (PPV) and negative predictive value (NPV) of ${ }^{18} \mathrm{~F}$-FDG-PET/CT in the detection of recurrence 
TABLE 2. ${ }^{18}$ F-FDG-PET/CT results, sensitivity, specificity, positive predictive value (PPV) and negative predictive value (NPV) according to final histopathologic diagnosis

\begin{tabular}{lccccccc}
\hline \multirow{2}{*}{ Histopathologic diagnosis } & \multicolumn{7}{c}{ 18F-FDG-PET/CT Results } \\
\cline { 2 - 8 } & Positive & Negative & Sensitivity & Specificity & PPV & NPV & Total (n) \\
\hline Malignant & TP $=24$ & FN $=0$ & & & & 24 \\
Benign & FP $=14$ & TN $=15$ & & & & 29 \\
Total $(\mathrm{n})$ & 38 & 15 & $100 \%$ & $51.7 \%$ & $63.1 \%$ & $100 \%$ & 53 \\
\hline
\end{tabular}

FN = False negative; FP = False positive $; \mathrm{TN}=$ True negative; $\mathrm{TP}=$ True positive

and/or metastasis were $100 \%, 51.7 \%, 63.1 \%$ and $100 \%$, respectively. ${ }^{18} \mathrm{~F}-\mathrm{FDG}$-PET/CT results and final histopathologic diagnosis were represented in Table 2.

${ }^{18} \mathrm{~F}-\mathrm{FDG}-\mathrm{PET} / \mathrm{CT}$ was truely positive in $48 \%$ of patients with normal Ca 19-9 and/or CEA levels and truely negative in $10 \%$ of cases with elevated Ca 19-9 and/or CEA levels according to histopathological confirmation or colonoscopy findings. In the follow-up, conventional imaging tests (CT or MRI) detected suspicious malignancy in $32 \%$ of the patients (17/53) and further examination with ${ }^{18} \mathrm{~F}-\mathrm{FDG}-\mathrm{PET} / \mathrm{CT}$ was truely negative in $35 \%$ of these cases (6/17) according to histopathology. ${ }^{18}$ F-FDG-PET/CT findings in histopathologically proven recurrence according to tumor markers $(\mathrm{Ca}$ 19-9 and/or CEA) and conventional imaging modalities (CT-MRI) were described in Table 3,4.

Mean SUVmax was $6.9 \pm 5.6(1-22)$ in benign group and $12.7 \pm 6.1$ (3.6-24) in malignant group. There is a statistically significant difference $(\mathrm{p}=$ 0.008 ) between them according to SUVmax. A boxplot graph illustrates the distribution of SUVmax in benign conditions versus disease recurrence

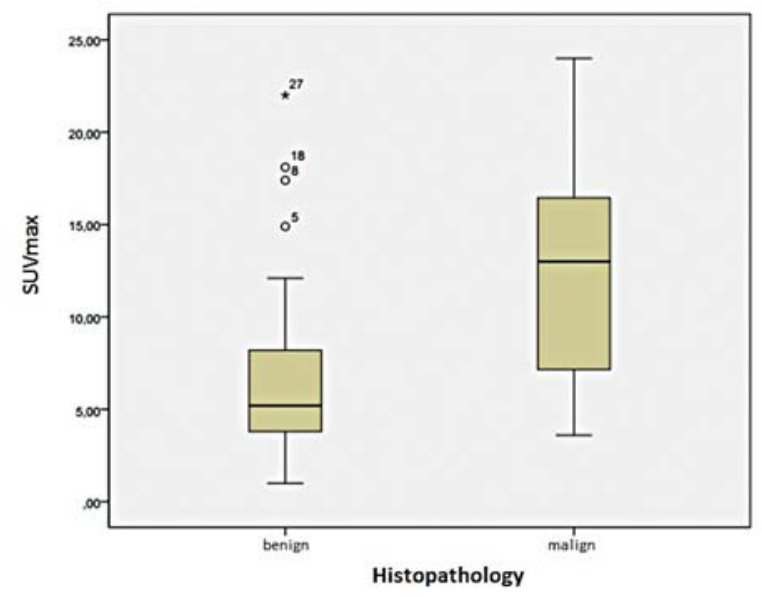

FIGURE 1. Box-plot graph illustrating the distribution of SUVmax through benign conditions and disease recurrence.
(Figure 1). ROC curve of SUVmax was plotted for the differentiation between benign conditions and malignant group (Area Under Curve: 0.755) (Figure 2). Neverthless, sensitivity and specificity couldn't be calculated due to undersampling and inconvenient SUVmax data not suggesting a determinant cutoff value. There is also a statistically significant difference between early and delayed SUVmax values of both groups separately in them $(p=0.013$ for benign group, $p=0.012$ for malignant group). However, we don't see a significant difference between them according to early and delayed SUVmax values $(p=0.238)$. Mean TLG of malignant group was $401 \pm 226$. Mean TLG of benign group was $148 \pm 126$ (median value: 44 ). The most important and striking statistical difference between them was found for TLG $(p=0.001)$.

\section{Discussion}

Recurrent disease is seen in $30-50 \%$ of patients with CRC after curative resection. ${ }^{6}$ The recurrence rate

TABLE 3. FDG-PET/CT findings according to serum Ca 19-9 or CEA levels for histopathologically proven recurrence

\begin{tabular}{lccc}
\hline \multicolumn{4}{c}{ 18F-FDG-PET/CT results } \\
\hline Ca 19-9 or CEA levels & True positive & True negative & Total $(\mathrm{n})$ \\
Elevated & 9 & 1 & 10 \\
Normal & 13 & 14 & 27 \\
Total $(\mathrm{n})$ & 22 & 15 & 37 \\
\hline
\end{tabular}

TABLE 4. Overlap between ${ }^{18} \mathrm{~F}-\mathrm{FDG}-\mathrm{PET} / \mathrm{CT}$ findings and conventional imaging modalities (CT or MRI) in histopathologically proven recurrence

\begin{tabular}{lccc}
\hline \multicolumn{4}{c}{ 18F-FDG-PET/CT results } \\
\hline CT/MRI & True positive & True negative & Total $(\mathrm{n})$ \\
Malign & 11 & 6 & 17 \\
\hline
\end{tabular}




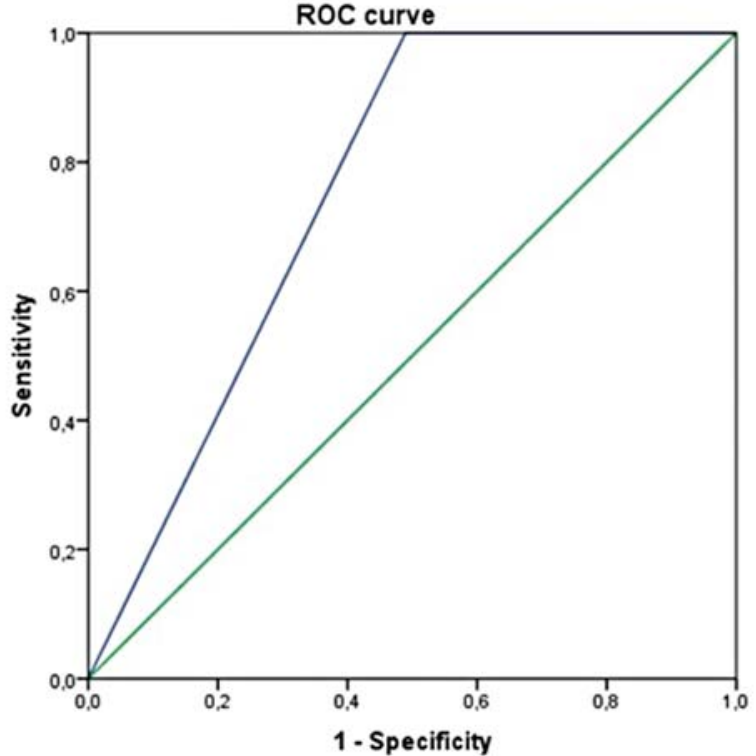

FIGURE 2. ROC curve drawn to indicate the detection and diagnostic accuracy of SUVmax in recurrence/metastasis.

in our study was $45 \%$ and it is accordant with literature. The main goal of follow-up surveillance is to reveal recurrences as soon as possible at an early stage for an immediate cure. ${ }^{5}$ Most of the relapsed cases are not operable at the time of diagnosis and $1 / 3$ of the patients with isolated locoregional or distant metastases survive 5 years. ${ }^{15}$
Several studies have proven that ${ }^{18} \mathrm{~F}$-FDG-PET/ $\mathrm{CT}$ is very sensitive, but not that much specific for detection of recurrence in CRC and affects patient management. ${ }^{3}$ Sobhani et al. ${ }^{16}$ included 130 patients with recurrent CRC in their study and compared ${ }^{18} \mathrm{~F}-\mathrm{FDG}$-PET/CT and conventional follow-up. They found no difference in recurrence rate, but recurrences were detected earlier by ${ }^{18} \mathrm{~F}-\mathrm{FDG}$-PET/ CT. Scott et al. ${ }^{17}$ showed that ${ }^{18}$ F-FDG-PET/CT detected 45 additional lesions in a multicenter prospective trial of 93 patients. Lu et al. ${ }^{18}$ studied sensitivity and specificity of diagnostic CT in a metaanalysis and found $51 \%$ and $90 \%$, respectively. The sensitivity and specificity of conventional imaging tests (CT or MRI) and ${ }^{18} \mathrm{~F}-\mathrm{FDG}-\mathrm{PET} / \mathrm{CT}$ were $71 \%$, $87 \%$ and $100 \%, 52 \%$, respectively in our study. In Figure 3, example of a true positive recurrent lesion suspected on MRI and confirmed by ${ }^{18} \mathrm{~F}-\mathrm{FDG}-$ PET/CT is shown. Maas et al. ${ }^{19}$, in a meta-analysis of 14 studies, investigated which imaging modality has the highest accuracy in CRC recurrence/metastasis suspected clinically or because of the elevated tumor marker levels. They reached the conclusion that ${ }^{18} \mathrm{~F}-\mathrm{FDG}$-PET/CT is absolutely more accurate (areas under curves were 0.94 for PET, 0.94 for PET/CT, 0.83 for CT). We found true positivity on ${ }^{18} \mathrm{~F}-\mathrm{FDG}$-PET/CT in $48 \%$ of patients with normal Ca 19-9 and/or CEA levels, although there was true negativity in $10 \%$ of cases with elevated Ca 19-9
(A)

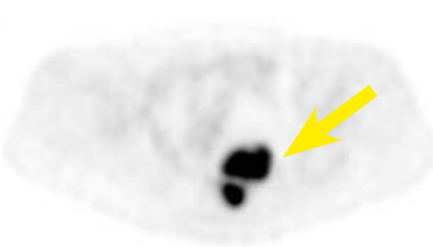

(D)

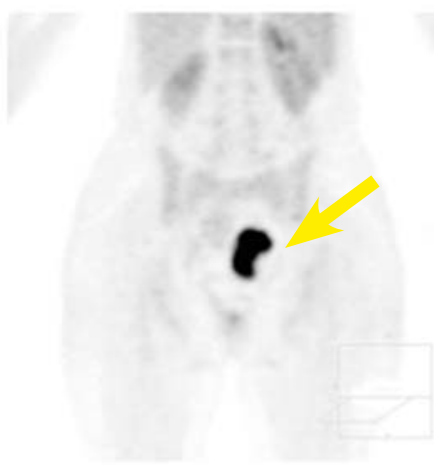

(B)
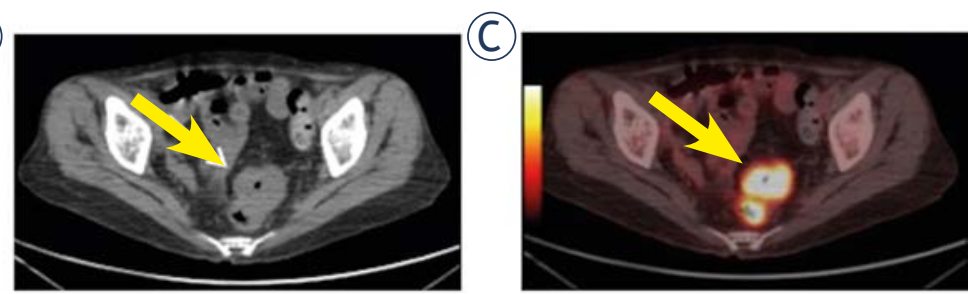

(E)

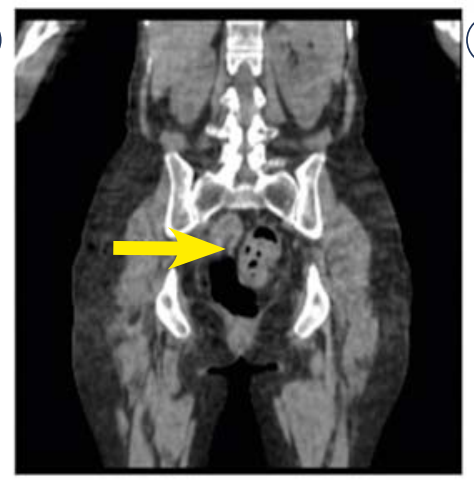

(2)

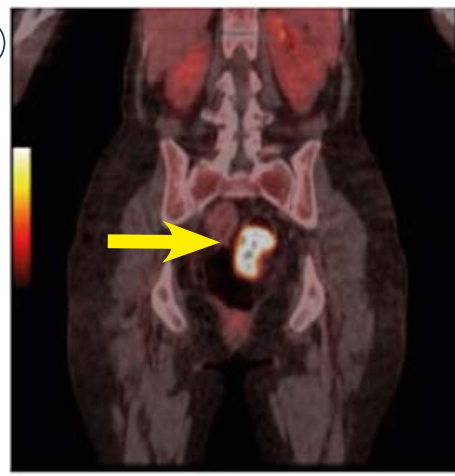

FIGURE 3. A female patient aged 51 years with rectum cancer was operated and treated by chemoradiotherapy. MRI findings revealed suspected metastasis with serum CEA and Ca 19-9 in normal range during the follow-up. Her axial PET (A), CT (B), fusion (C) and coronal PET (D), CT (E), fusion (F) images on ${ }^{18} \mathrm{~F}-\mathrm{FDG}-\mathrm{PET} / \mathrm{CT}$ showed a focal uptake in sigmoid colon with a SUVmax of 13.7 and TLG of 272 accompanied by wall thickening causing a mass lesion on CT component (arrows). Histopathology established the diagnosis as recurrence. 
and/or CEA levels. All our findings are in line with data from literature.

Although functional imaging with ${ }^{18} \mathrm{~F}$-FDG PET is a useful technique for evaluating treatment response, it has some limitations. ${ }^{18} \mathrm{~F}-\mathrm{FDG}$ is taken up at a relatively higher rate in cancer cells and accumulates during glucose metabolism. However, cancer cells are not the only cells that are metabolically hyperactive. Inflammation, infection and other non-neoplastic conditions such as hyperplastic colorectal polyps may have increased FDG accumulation. ${ }^{20,21}$ Depending upon this, PET scans have a high sensitivity but a low specificity for $\mathrm{CRC}^{21}$ as it was also the case in our study. Compared to PET alone, combination of PET and CT, having the advantage of detecting metabolic abnormality with anatomic localization, is superior in localizing lesions and differentiating between physiologic and malignant uptake of FDG. The benign pathologies diagnosed in our study were granulation tissue, fibrin and inflammation, fibrosis, pyelonephritis, ulceration of colonic mucosa, fibrosis and inflammation, polip, as well as changes secondary to radiotherapy or operation.

Some manipulative registration or intervention methods on ${ }^{18} \mathrm{~F}-\mathrm{FDG}-\mathrm{PET} / \mathrm{CT}$ imaging have been suggested to increase the specificity. Recently, Prieto et al. ${ }^{22}$ suggested voxel-based dual-time ${ }^{18} \mathrm{~F}$-FDG-PET images for brain tumors and demonstrated that parametric images provided enhanced tumor identification. Voxel-based analysis is different from VOIbased one in that it is an attempt enabling calculation of real increase in delayed scan of the same voxel. Choi et al. ${ }^{12}$ investigated voxel-based dualtime ${ }^{18}$ F-FDG-PET parametric imaging in the evaluation of residual tumor for rectal cancer and found promising results. Dual-time ${ }^{18} F$-FDG-PET has also been reported to improve diagnostic accuracy for several cancers. ${ }^{14}$

\section{(B)}

This is based on the different pattern of FDG uptake in malignant tumors displaying a slow increment, whereas benign lesions show an earlier peak and then a decline. ${ }^{23}$ Lots of benign conditions and physiologic FDG uptakes displaying focal or diffuse FDG accumulations in gastrointestinal tract can be seen in patients with CRC during the follow-up and may be confused with true pathologic lesions. When such a pattern is observed on ${ }^{18} \mathrm{~F}-\mathrm{FDG}-\mathrm{PET} / \mathrm{CT}$, invasive interventions (colonoscopy, biopsy) are recommended. So it is essential to distinguish them. An example of false positivity is shown in Figure 4.

Miyake et al. ${ }^{24}$ showed that delayed scans increased correct differentiation of physiologic FDG uptakes causing false positivities from foci of pathologic uptake in CRC. Yoon et al. ${ }^{25}$ reported that dual-time ${ }^{18} \mathrm{~F}-\mathrm{FDG}-\mathrm{PET} / \mathrm{CT}$ had better accuracy in diagnosing tumor response and recurrence. We researched the difference of SUVmax on early and delayed images of dual-time ${ }^{18} \mathrm{~F}-\mathrm{FDG}$-PET between
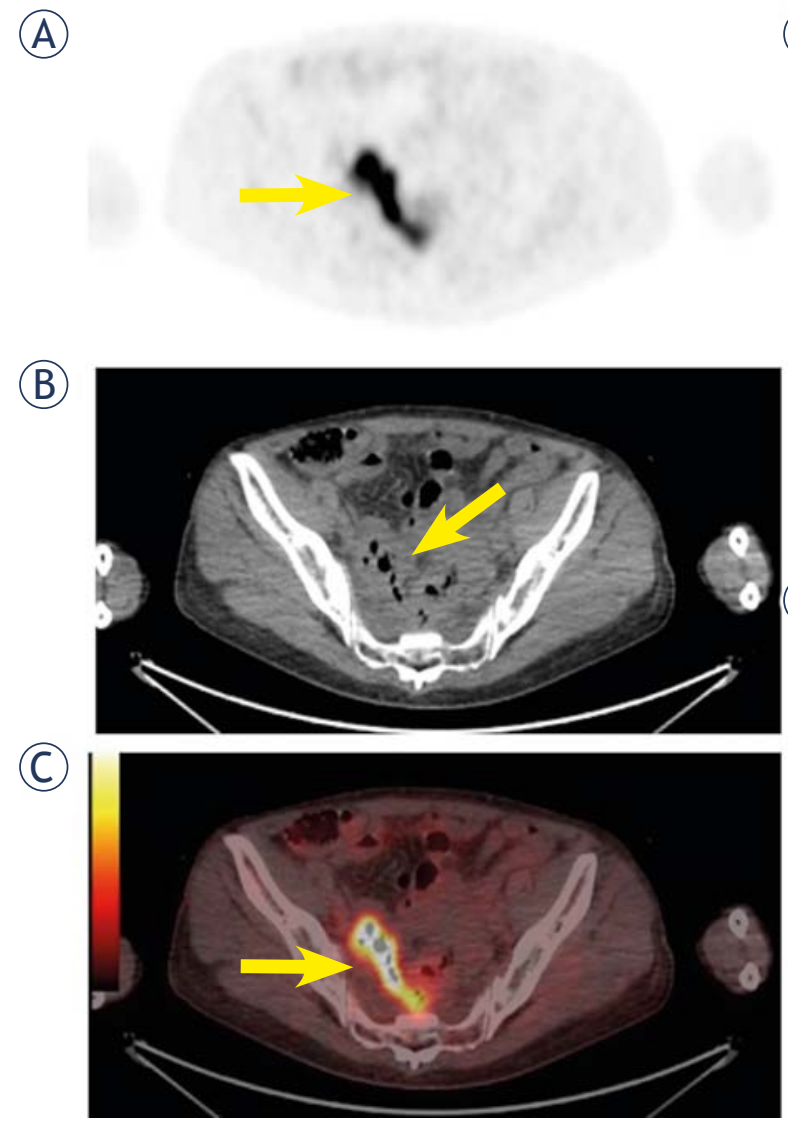

(1)

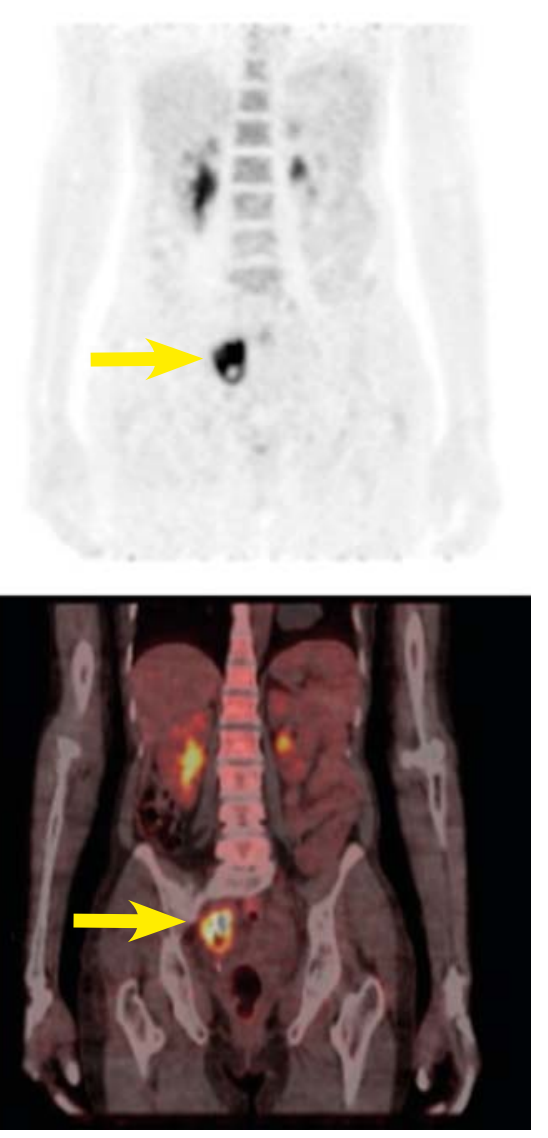

FIGURE 4. A female patient aged 73 years with sigmoid colon cancer was operated and treated by chemoradiotherapy. Her serum CEA level was $3.2 \mathrm{ng} / \mathrm{ml}$, and Ca 19-9 was $7.3 \mathrm{U} / \mathrm{ml}$. In the evaluation of treatment response; axial PET (A), CT (B), fusion (C) and coronal PET (D), fusion (E) images on ${ }^{18}$ F-FDG-PET/CT exhibited a diffuse uptake in sigmoid colon with a SUVmax of 10.1 and TLG of 154 accompanied by wall thickening on CT component (arrows). This uptake raised the suspicion of a probable recurrence, but histopathology confirmed it as benign. 
benign and malignant group. We didn't find a meaningful statistics and discriminative power for this parameter.

We investigated the value of SUVmax and TLG too. Most of the studies including SUVmax and TLG evaluation in CRC are related to prognosis estimation. Marcus et al. ${ }^{26}$ declared SUVmax and TLG to be higher in patients having bad prognosis. Gade et al. ${ }^{2}$ found a lower mean SUVmax of 8.6, Marcus et al. ${ }^{26} 7.3$ in recurrent CRC when compared to ours (12.7). Shamim et al. ${ }^{27}$ found a significant increase according to mean SUVmax in recurrence (11.8 for recurrence versus 3.7 for benign conditions) in a study of 32 patients with CRC. They were 12.7 for recurrence against 6.9 for benign group in our study and there was a significant difference $(\mathrm{p}=$ 0.008). Giacomobono et al. ${ }^{28}$ assessed SUVmax in CRC patients with increased CEA levels and found a worse overall survival for SUVmax values greater than 5.7. Inflammatory pathologies, fibrosis or edema following irradiation and/or surgery can also be FDG-avid and their SUVmax values can be as high as malignant ones due to the degree of cellular metabolism reflected by ${ }^{18} \mathrm{~F}-\mathrm{FDG}$ uptake. ${ }^{11,12}$ Therefore, big overlaps in SUVmax values of both benign conditions and recurrent/metastatic disease may be seen just like in our study. However, we found SUVmax was very helpful for the differentiation of recurrent disease from benign conditions. As our results trended towards improved diagnostic accuracy, a determinative cutoff value for SUVmax was not obtained. Undersampling and inconvenient SUVmax data didn't let an estimated cutoff value on ROC curve for related sensitivity and specificity calculation.

Quantitative PET parameters have been used in prognosis estimation and evaluation of treatment response for several cancers. There is not a specific study in literature which investigated metabolic tumor parameters for the differentiation of benign conditions from recurrence. As far as we know, our study is the first one in literature. Higher metabolic activity and glucose consumption of tumor cells are measured by these parameters. SUVmax is the first one and represents the highest FDG uptake within the tumor. More lately volume-based metabolic parameters emerged out. TLG is a volume-based metabolic tumor parameter having widespread use and increasing recognition in many cancers as a predictor. Arslan et al. ${ }^{29}$ reported it has a role in prediction of survival for patients with small cell lung cancer. Caglar et al. ${ }^{30}$ investigated metabolic tumor markers in recurrent CRC and found moderate correlation. Marcus et al. ${ }^{26}$ found mean TLG
280, Caglar et al. ${ }^{30} 55$ in recurrent CRC. It was 401 in our study. There was significant difference between benign conditions and recurrence according to mean TLG and TLG was the most striking factor for this purpose in the study $(p=0.001)$. Again, we didn't intentionally calculate a cutoff value which could be misleading due to insufficient sampling. But first impressions imply determinative cutoff values for SUVmax and TLG increasing the specificity may be obtained from studies with larger patient numbers. Higher SUVmax and TLG values in suspected cases would favour in recurrence/metastasis and support the necessary invasive interventions.

Absence of an estimated cutoff value on ROC curve for related sensitivity, specificity calculations due to undersampling and inconvenient SUVmax data was a limitation in the study. The number of subjects were small. Evaluation with larger populations is required for definitive results. Study design was also a limitation. Ideally, prospective studies are needed. The other limitation was that TLG calculations and dual-time imaging could not be performed for all the patients.

\section{Conclusions}

FDG uptake on PET/CT imaging is quite sensitive for both benign and malignant lesions in patients with CRC. ${ }^{18} \mathrm{~F}-\mathrm{FDG}-\mathrm{PET} / \mathrm{CT}$ appears to be very beneficial in revealing especially true-negative lesions suspected of recurrence or metastasis and may prevent unnecessary treatments. Although SUVmax is a strong metabolic parameter $(p=0.008)$, TLG seems to be the best predictor in recurrence of colorectal cancers $(p=0.001)$. Both are increasing the specificity of ${ }^{18} \mathrm{~F}-\mathrm{FDG}-\mathrm{PET} / \mathrm{CT}$.

\section{References}

1. Hammond K, Margolin DA. The role of postoperative surveillance in colorectal cancer. Clin Colon Rectal Surg 2007; 20: 249-54. doi: 10.1055/s-2007984869

2. Gade $M$, Kubik $M$, Fisker RV Thorlacius-Ussing $O$, Petersen $U$. Diagnostic value of (18)F-FDG PET/CT as first choice in the detection of recurrent colorectal cancer due to rising CEA. Cancer Imaging 2015; 15: 11. doi: 10.1186/ s40644-015-0048-y

3. Laurens ST, Oyen WJ. Impact of fluorodeoxyglucose PET/Computed Tomography on the management of patients with colorectal cancer. PET Clin 2015; 10: 345-60. doi: 10.1016/j.cpet.2015.03.007

4. Chen CH, Hsieh MC, Lai CC, Yeh CY, Chen JS, Hsieh PS, et al. Lead time of carcinoembryonic antigen elevation in the postoperative follow-up of colorectal cancer did not affect the survival rate after recurrence. Int $J$ Colorectal Dis 2010; 25: 567-71. doi: 10.1007/s00384-010-0889-6 
5. Chiaravalloti A, Fiorentini A, Palombo E, Rinino D, Lacanfora A, Danieli R, et al. Evaluation of recurrent disease in the re-staging of colorectal cancer by 18F-FDG PET/CT: Use of CEA and CA 19-9 in patient selection. Oncol Lett 2016; 12: 4209-13. doi: 10.3892/ol.2016.5143

6. Van de Velde CJ, Boelens PG, Borras JM, Coebergh JW, Cervantes A, Blomqvist L, et al. EURECCA colorectal: Multidisciplinary management: European consensus conference colon \& rectum. Eur J Cancer 2014; 50: 1.e1-34. doi: 10.1016/j.ejca.2013.06.048

7. Tan E, Gouvas N, Nicholls RJ, Ziprin P, Xynos E, Tekkis PP. Diagnostic precision of carcinoembryonic antigen in the detection of recurrence of colorectal cancer. Surg Oncol 2009; 18: 15-24. doi: 10.1016/j.suronc.2008.05.008

8. Filella X, Molina R, Piqué JM, Garcia-Valdecasas JC, Grau JJ, Novell F, et al. Use of CA 19-9 in the early detection of recurrences in colorectal cancer: Comparison with CEA. Tumour Biol 1994; 15: 1-6.

9. Panagiotidis E, Datseris IE, Rondogianni P, Vlontzou E, Skilakaki M, Exarhos D, et al. Does CEA and CA 19-9 combined increase the likelihood of 18F-FDG in detecting recurrence in colorectal patients with negative CeCT? NuCl Med Commun 2014; 35: 598-605. doi: 10.1097/MNM.0000000000000107

10. Sanli Y, Kuyumcu S, Ozkan ZG, Kilic L, Balik E, Turkmen C, et al. The utility of FDG-PET/CT as an effective tool for detecting recurrent colorectal cancer regardless of serum CEA levels. Ann Nucl Med 2012; 26: 551-8. doi: 10.1007/ s12149-012-0609-0

11. Zhuang $\mathrm{H}$, Alavi A. 18-Fluorodeoxyglucose positron emission tomographic imaging in the detection and monitoring of infection and inflammation. Semin Nucl Med 2002; 32: 47-59. doi: 10.1053/snuc.2002.29278

12. Choi H, Yoon HJ, Kim TS, Oh JH, Kim DY, Kim SK. Voxel-based dual-time 18F-FDG parametric imaging for rectal cancer: differentiation of residual tumor from postchemoradiotherapy changes. Nucl Med Commun 2013; 34: 1166-73. doi: 10.1097/MNM.0000000000000002

13. Chan K, Welch S, Walker-Dilks C, Raifu A. Ontario provincial Gastrointestinal Disease Site Group. Evidence-based guideline recommendations on the use of positron emission tomography imaging in colorectal cancer. Clin Oncol ( $R$ Coll Radiol) 2012; 24: 232-49. doi: 10.1016/j.clon.2011.11.008

14. Basu S, Alavi A. Partial volume correction of standardized uptake values and the dual time point in FDG-PET imaging: should these be routinely employed in assessing patients with cancer? Eur J Nucl Med Mol Imaging 2007; 34: 1527-9. doi: 10.1007/s00259-007-0467-5

15. Bowne WB, Lee B, Wong WD, Ben-Porat L, Shia J, Cohen AM, et al. Operative salvage for locoregional recurrent colon cancer after curative resection: An analysis of 100 cases. Dis Colon Rectum 2005; 48: 897-909. doi: 10.1007/ s10350-004-0881-8

16. Sobhani I, Tiret E, Lebtahi R, Aparicio T, Itti E, Montravers F, et al. Early detection of recurrence by 18FDG-PET in the follow-up of patients with colorecta cancer. Br J Cancer 2008; 98: 875-80. doi: 10.1038/sj.bjc.6604263

17. Scott AM, Gunawardana DH, Kelley B, Stuckey JG, Byrne AJ, Ramshaw JE, et al. PET changes management and improves prognostic stratification in patients with recurrent colorectal cancer: results of a multicenter prospective study. J Nucl Med 2008; 49: 1451-7. doi: 10.2967/jnumed.108.051615

18. Lu YY, Chen JH, Chien CR, Chen WT, Tsai SC, Lin WY, et al. Use of FDG-PET or $\mathrm{PET} / \mathrm{CT}$ to detect recurrent colorectal cancer in patients with elevated CEA: systematic review and meta-analysis. Int J Colorectal Dis 2013; 28: 1039-47. doi: $10.1007 /$ s00384-013-1659-z

19. Maas M, Rutten IJ, Nelemans PJ, Lambregts DM, Cappendijk VC, Beets GL, et al. What is the most accurate whole-body imaging modality for assessment of local and distant recurrent disease in colorectal cancer? A meta-analysis: imaging for recurrent colorectal cancer. Eur J Nucl Med Mol Imaging 2011 38: 1560-71. doi: 10.1007/s00259-011-1785-1

20. Shmidt $E$, Nehra $V$, Lowe $V$, Oxentenko AS. Clinical significance of incidental [18F]FDG uptake in the gastrointestinal tract on PET/CT imaging: a retrospective cohort study. BMC Gastroenterol 2016; 16: 125. doi: 10.1186/ s12876-016-0545-x

21. Abdel-Nabi H, Doerr RJ, Lamonica DM, Cronin VR, Galantowicz PJ, Carbone $\mathrm{GM}$, et al. Staging of primary colorectal carcinomas with fluorine-18 fluorodeoxyglucose whole-body PET: correlation with histopathologic and CT findings. Radiology 1998; 206: 755-60. doi: 10.1148/radiology.206.3.9494497

22. Prieto E, Marti-Climent JM, Dominguez-Prado I, Garrastachu P, Díez-Valle R, Tejada S, et al. Voxel-based analysis of dual-time-point 18F-FDG PET images for brain tumor identification and delineation. J Nucl Med 2011; 52: 865-72. doi: 10.2967/jnumed.110.085324
23. Schillaci O. Use of dual-point fluorodeoxyglucose imaging to enhance sensitivity and specificity. Semin Nucl Med 2012; 42: 267-80. doi: 10.1053/j. semnuclmed.2012.02.003

24. Miyake KK, Nakamoto Y, Togashi K. Dual-time-point 18F-FDG PET/CT in patients with colorectal cancer: clinical value of early delayed scanning. Ann Nucl Med 2012; 26: 492-500. doi: 10.1007/s12149-012-0599-y

25. Yoon HJ, Kim SK, Kim TS, et al. New application of dual time point 18F-FDG $\mathrm{PET} / \mathrm{CT}$ in the evaluation of neoadjuvant chemoradiation response of locally advanced rectal cancer. Clin Nucl Med 2013; 38: 7-12. doi: 10.1097/ RLU.0b013e3182639a58

26. Marcus C, Wray R, Taghipour M, Marashdeh W, Ahn SJ, Mena E, et al. Journal club: Value of quantitative FDG PET/CT volumetric biomarkers in recurrent colorectal cancer patient survival. AJR Am J Roentgenol 2016; 207: 257-65. doi: 10.2214/AJR.15.15806

27. Shamim SA, Kumar R, Halanaik D, Shandal V, Reddy RM, Bal CS, et al. Role of FDG-PET/CT in detection of recurrent disease in colorectal cancer. Nucl Med Commun 2010; 31: 590-6. doi: 10.1097/MNM.0b013e328338a120

28. Giacomobono S, Gallicchio R, Capacchione D, Nardelli A, Gattozzi D, Lettini G, et al. F-18 FDG PET/CT in the assessment of patients with unexplained CEA rise after surgical curative resection for colorectal cancer. Int $\mathrm{J}$ Colorectal Dis 2013; 28: 1699-705. doi: 10.1007/s00384-013-1747-0.

29. Arslan N, Tuncel M, Kuzhan O, Alagoz E, Budakoglu B, Ozet A, et al. Evaluation of outcome prediction and disease extension by quantitative 2-deoxy2-[18F] fluoro-D-glucose with positron emission tomography in patients with small cell lung cancer. Ann Nucl Med 2011; 25: 406-13. doi: 10.1007/ s12149-011-0478-y

30. Caglar M, Yener C, Karabulut E. Value of CT, FDG PET-CT and serum tumor markers in staging recurrent colorectal cancer. Int J Comput Assist Radiol Surg 2015; 10: 993-1002. doi: 10.1007/s11548-014-1115-8 